\title{
CBS versus GLS stabilization of the incompressible Navier-Stokes equations and the role of the time step as stabilization parameter
}

\author{
R. Codina ${ }^{1, *}$ and O. C. Zienkiewicz ${ }^{2}$ \\ ${ }^{1}$ Departament de Resistència de Materials i Estructures a l'Enginyeria, Universitat Politècnica de Catalunya, \\ Jordi Girona 1-3, Edifici C1, 08034 Barcelona, Spain \\ ${ }^{2}$ Institute of Numerical Methods in Engineering, University College of Swansea, Swansea SA2 8PP, U.K.
}

\begin{abstract}
SUMMARY
In this work we compare two apparently different stabilization procedures for the finite element approximation of the incompressible Navier-Stokes equations. The first is the characteristic-based split (CBS). It combines the characteristic Galerkin method to deal with convection dominated flows with a classical splitting technique, which in some cases allows us to use equal velocity-pressure interpolations. The second approach is the Galerkin-least-squares (GLS) method, in which a least-squares form of the element residual is added to the basic Galerkin equations. It is shown that both formulations display similar stabilization mechanisms, provided the stabilization parameter of the GLS method is identified with the time step of the CBS approach. This identification can be understood from a formal Fourier analysis of the linearized problem. Copyright (C) 2001 John Wiley \& Sons, Ltd.
\end{abstract}

KEY WORDS: characteristic scheme; fractional step methods; stabilization; GLS

\section{INTRODUCTION}

The Navier-Stokes equations for an incompressible fluid moving in a domain $\Omega$ during the time interval $] 0, T[$ can be written as

$$
\begin{aligned}
\partial_{t} \mathbf{u}+\mathbf{u} \cdot \nabla \mathbf{u}-v \Delta \mathbf{u}+\nabla p & =\mathbf{f} \\
\nabla \cdot \mathbf{u} & =\mathbf{0}
\end{aligned}
$$

where $\mathbf{u}$ is the velocity, $p$ the pressure, $\mathbf{f}$ the vector of body forces and $v$ the kinematic viscosity. These equations have to be supplied with an initial condition of the form $\mathbf{u}=\mathbf{u}_{0}$ at $t=0$ and a boundary condition which, for simplicity, will be taken as $\mathbf{u}=\mathbf{0}$ on $\partial \Omega$.

\footnotetext{
*Correspondence to: R. Codina, Departament de Resistència de Materials i Estructures a 1'Enginyeria, Universitat Politècnica de Catalunya, Jordi Girona 1-3, Edifici C1, 08034 Barcelona, Spain
} 
There are several ways to deal with the space and time approximation of this problem. When the standard Galerkin method is used, the most common is the so-called method of lines, in which space is discretized first and the resulting system of ordinary differential equations (with an algebraic constraint) is integrated in time using a finite difference scheme. Another possibility is to use a space-time finite element interpolation.

Whichever the time discretization method is, the spatial discretization may suffer from two major numerical problems. The first is the velocity-pressure interpolation. The zero divergence restriction imposes a compatibility condition for this interpolation that, in particular, prevents the use of equal interpolation. Likewise, when convection dominates, spurious spatial oscillations may occur. This has to be understood as a numerical problem, and is independent of the complicated flow features (including turbulence) that appear when the Reynolds number is high. The parameter that plays a role in this case is the cell Reynolds number rather than the global one. See Reference [1] for background.

These two numerical problems have been treated with a variety of finite element formulations. The objective of this paper is precisely to compare two of them, namely, the characteristic-based split (CBS) [2-4] and the Galerkin-least-squares (GLS) [5,6] methods. The idea of the first is to stabilize convection by using a finite difference discretization along the characteristics, and to rely on the stabilizing effect on the pressure of a classical splitting technique. On the other hand, the GLS method provides control on the element residual of

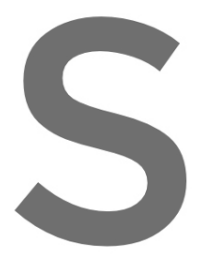
the momentum equation, terpolation. In the case space-time finite ele here.

In this paper, we summarize the basic developments behind th and show that they introduce similar stabilizing terms. The
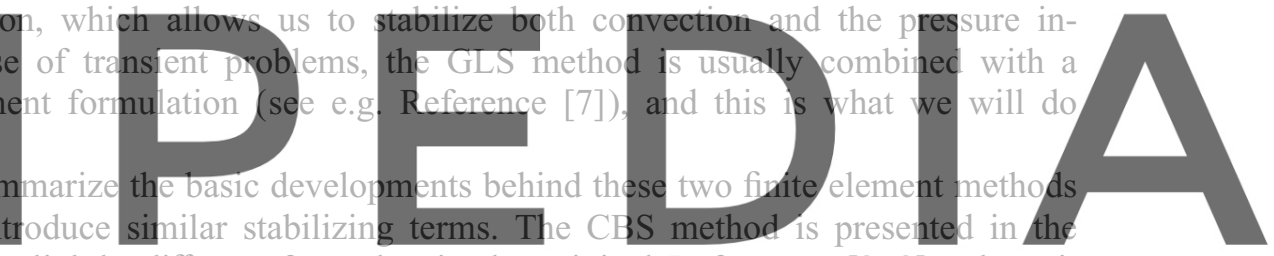

following section in a slightly different form that in the original References [2, 3], where it

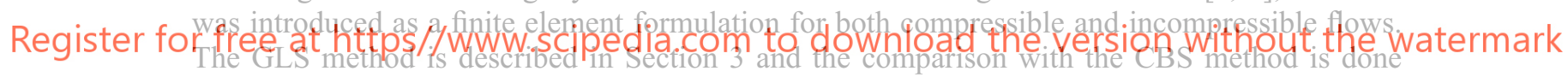

in Section 4. Section 5 presents a discussion about the relationship between the stabilizing parameters and the time step size. Finaliy, we draw some conciusions.

\section{THE CHARACTERISTIC-BASED SPLITTING (CBS) METHOD}

\subsection{The characteristic Galerkin method revisited}

Let us denote by $\mathbf{X}(\overline{\mathbf{x}}, \bar{t} ; t)$ the trajectory of the particle that at time $t=\bar{t}$ is located at the spatial point $\overline{\mathbf{x}}$, so that $\mathbf{X}(\overline{\mathbf{x}}, \bar{t} ; \bar{t})=\overline{\mathbf{x}}$. This trajectory will be the solution of the problem

$$
\begin{aligned}
\frac{\mathrm{d}}{\mathrm{d} t} X_{i}(t) & =u_{i}(\mathbf{X}(t), t) \\
X_{i}(\bar{t}) & =\bar{x}_{i}
\end{aligned}
$$

for $i=1,2,3$ (in 3D). In the short-hand notation $\mathbf{X}(t)$ it is understood that $\mathbf{X}$ depends also on $\bar{t}$ and $\overline{\mathbf{x}}$ through the initial condition (4). 
The conservation of momentum and mass for a particle of incompressible fluid as it moves along its trajectory may be then written as

$$
\begin{aligned}
\frac{\mathrm{d}}{\mathrm{d} t} \mathbf{u}(\mathbf{X}(t), t)-v \Delta \mathbf{u}(\mathbf{X}(t), t)+\nabla p(\mathbf{X}(t), t) & =\mathbf{f}(\mathbf{X}(t), t) \\
\nabla \cdot \mathbf{u}(\mathbf{X}(t), t) & =0
\end{aligned}
$$

Equations (5) and (6) for $t=\bar{t}$ are precisely the Navier-Stokes equations (1) and (2) at the spatial point $\mathbf{x}=\overline{\mathbf{x}}$ and at time $t=\bar{t}$. We shall use $\mathbf{x}$ instead of $\overline{\mathbf{x}}$, to emphasize that this position is arbitrary.

The idea now is to discretize the derivative $\mathrm{d} / \mathrm{d} t$ in (5) using a finite difference scheme, that is, to discretize the total derivative in (1) along the characteristics. If we employ a single-step finite difference discretization, the highest time accuracy that we can obtain is second order. In order to be as accurate as possible in the first (spatial) argument of the velocity and the pressure, problem (5)-(6) has to be discretized up to second-order. Once the second-order time discretization is done, different schemes can be obtained by approximating the second (temporal) argument of the velocity and the pressure, yielding discrete schemes with a lower temporal accuracy but potentially second order accurate in space.

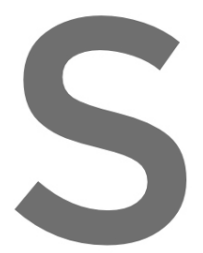

$$
\text { Consider a uniforn }
$$

that we have the solution at Let $\bar{t}$ be a certain tin the momentum equation. the generalized trapezoidal ru
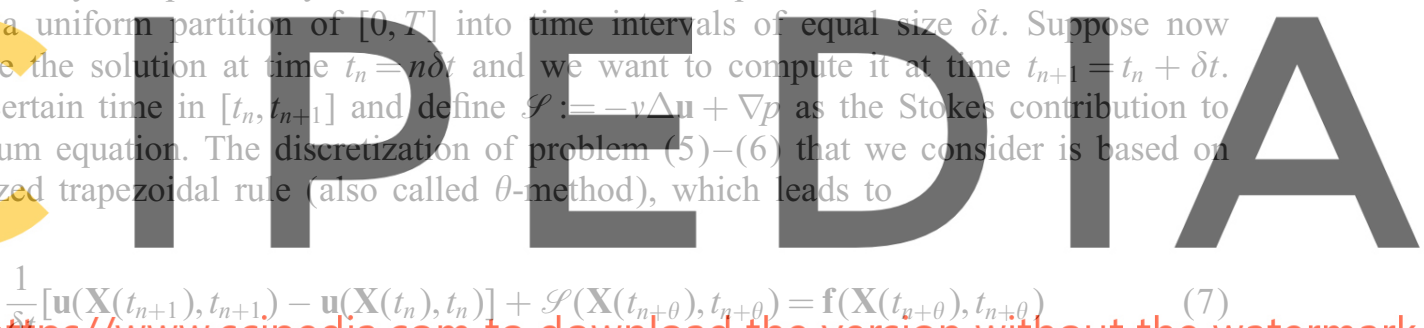

Register for free at hetps//WWW.scipedia.com to download the version without the watermark $\nabla \cdot \mathbf{u}(\mathbf{X}(\bar{t}), \bar{t})=0$

where $t_{n+\theta}=\theta t_{n+1}+(1-\theta) t_{n}$ and $\theta \in[0,1]$. For the reasons explained above, we must take $\theta=\frac{1}{2}$, since this is the only value of $\theta$ that yields second-order accuracy (Crank-Nicolson scheme).

Let $\varphi(\mathbf{x}, t)$ be a generic function. The distinctive feature of our approach is that we shall derive an explicit expression for $\varphi\left(\mathbf{X}\left(t_{n+1}\right), t_{n+1}\right)$ and $\varphi\left(\mathbf{X}\left(t_{n}\right), t_{n}\right)$ in terms of $\varphi^{n+1}:=\varphi\left(\mathbf{x}, t_{n+1}\right)$ and $\varphi^{n}:=\varphi\left(\mathbf{x}, t_{n}\right)$. The parameter that we have still free is the reference time $\bar{t}$. Within the time step $\left[t_{n}, t_{n+1}\right]$ we shall take this time as $\bar{t}=t_{n}+\gamma \delta t$, with $\gamma$ arbitrary (see Figure 1 ). Two particular cases of interest are $\gamma=\frac{1}{2}$ and $\gamma=1$, that is, $\bar{t}=t_{n}+\delta t / 2$ and $\bar{t}=t_{n+1}$. The former yields the classical Crank-Nicolson discretization of problem (1)-(2), whereas the latter introduces some additional terms than enhance the stability of the numerical scheme. From the geometrical standpoint, if $\bar{t}=t_{n}+\delta t / 2$ then (7) may be viewed as centred discretization along the characteristics. On the other hand, for $\bar{t}=t_{n+1}$ we move backwards.

For a same order of approximation, we shall use explicit approximations instead of implicit ones. Thus, other schemes apart from the one derived below exist with the same properties of accuracy. The results that follow are a summary of the developments presented in Reference [8]. 


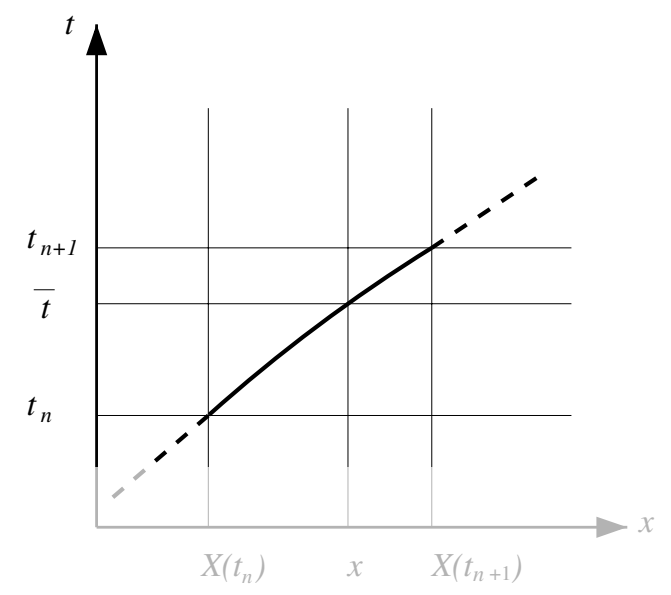

Figure 1. Discretization along the characteristics.

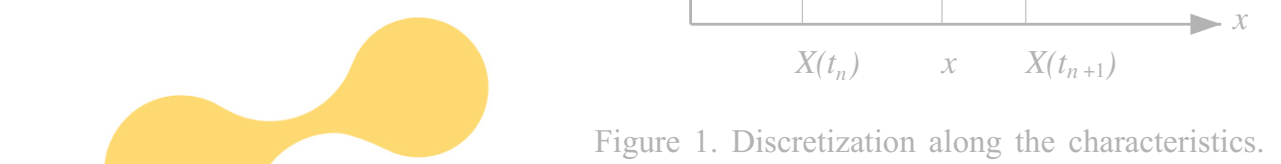

Expanding in Taylor series the solution of problem (3)-(4) and also using a Taylor
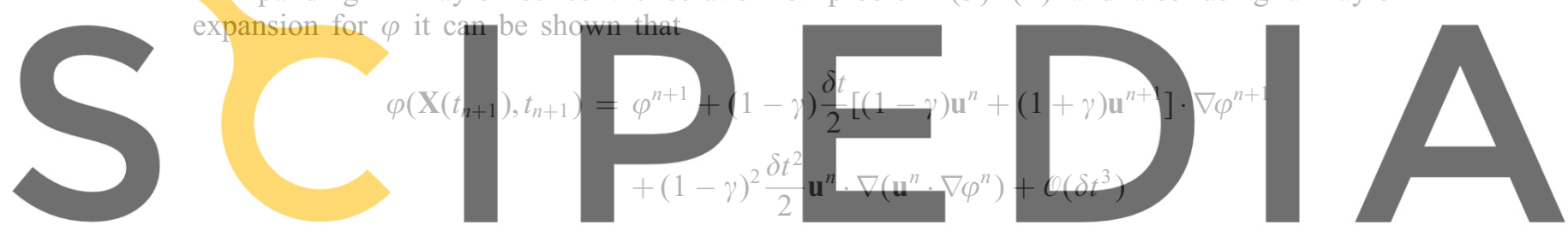

Register for free at https//www.scipedia.com to download the version without the watermark

$$
+\gamma^{2} \frac{\delta t^{2}}{2} \mathbf{u}^{n} \cdot \nabla\left(\mathbf{u}^{n} \cdot \nabla \varphi^{n}\right)+o\left(\delta t^{3}\right)
$$

These expressions are approximations to the function $\varphi$ along the trajectory of the particles (i.e. the characteristics of the total derivative operator) at the beginning and the end of the time interval $\left[t_{n}, t_{n+1}\right]$ under consideration. Using them, after several algebraic manipulations it is finally found that [8]

$$
\begin{gathered}
\frac{1}{\delta t}\left(\mathbf{u}^{n+1}-\mathbf{u}^{n}\right)+\mathbf{u}^{n+1 / 2} \cdot \nabla \mathbf{u}^{n+1-\gamma}-v \Delta \mathbf{u}^{n+1 / 2}+\nabla p^{n+1 / 2}-\mathbf{f}^{n+1 / 2} \\
-(2 \gamma-1) \frac{\delta t}{2} \mathbf{u}^{n} \cdot \nabla\left(\mathbf{u}^{n} \cdot \nabla \mathbf{u}^{n}-v \Delta \mathbf{u}^{n}+\nabla p^{n}-\mathbf{f}^{n}\right)=\mathbf{0} \\
\nabla \cdot \mathbf{u}^{n+1}=0
\end{gathered}
$$

Equation (10) a second-order approximation of the incompressibility constraint imposed at $\bar{t}=t_{n}+\gamma \delta t($ see $(6))$. Since $\nabla \cdot \mathbf{u}\left(\mathbf{x}, t_{n}+\gamma \delta t\right)=\gamma \nabla \cdot \mathbf{u}^{n+1}+(1-\gamma) \nabla \cdot \mathbf{u}^{n}+\mathcal{O}\left(\delta t^{2}\right)$, and, assuming the initial condition to be divergence free, (10) is enough to ensure that $\mathbf{u}\left(\mathbf{x}, t_{n}+\gamma \delta t\right)$ is also divergence free up to second order. 
It is observed from (9) that for $\gamma=\frac{1}{2}$ we obtain the classical Crank-Nicolson approximation of the original momentum Equation (1), even though (9)-(10) have been obtained from the discretization of problem (5)-(6). A posteriori we may, however, interpret them as the time discretization of problem (1)-(2) plus the introduction of the term $-\tau_{\mathrm{cg}} \mathbf{u}^{n} \cdot \nabla \mathbf{R}^{n}$, where

$$
\begin{aligned}
\tau_{\mathrm{cg}} & :=(2 \gamma-1) \frac{\delta t}{2} \\
\mathbf{R}^{n} & :=\mathbf{u}^{n} \cdot \nabla \mathbf{u}^{n}-v \Delta \mathbf{u}^{n}+\nabla p^{n}-\mathbf{f}^{n}
\end{aligned}
$$

From problem (9)-(10) we can obtain simplified versions of first-order accuracy in time by replacing quantities at intermediate times between $t_{n}$ and $t_{n+1}$ either by values at $t_{n}$ or $t_{n+1}$ (explicit or implicit versions, respectively). Observe that this only involves an approximation in time, not in space. Neglecting the terms affected by the time step size in (9)-(10) would imply also an approximation in space, since they come from an approximation of the characteristics $\mathbf{X}(t)$.

The characteristic Galerkin method in its original form $[9,10]$ was designed as a crude first-order approximation in time (backward Euler scheme) and taking $\gamma=1$ with our notation. It also has the drawback of having to integrate the trajectories at each time step and interpolating the value $\rho\left(\mathbf{X}\left(t_{n}\right), t_{n}\right)$ within the element to which the point $\mathbf{X}\left(t_{n}\right)$ belongs. The idea of approximating References $[3,11]$ and

2.2. Pressure splitting

The next ingredient of the CBS algorithm is a
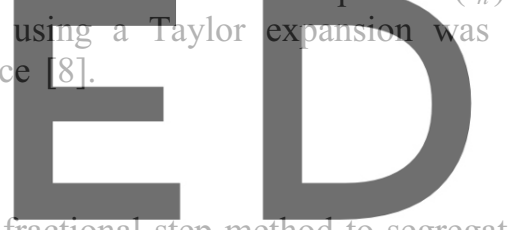

from the velocity calculation, in the spirit of the classical projection method $[12,13]$. The

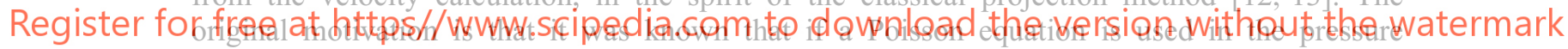

calculation, it is possible to use equal velocity-pressure interpolation, that is to say, it is not necessary to satisfy the classical inf-sup condition. This fact had been noticed in many works and it was heuristically justified in Reference [2]. However, we will not explore here the stabilization mechanism identified in Reference [2], but rather the approach noted in Reference [14]. The difference between both points of view is that while in Reference [2] we analyse the problem for the end-of-step velocity, in Reference [14] the author points out the stabilized problem of which the intermediate velocity is solution. Here, we pursue this viewpoint.

Let us consider a simplified, fully implicit first-order version of problem one (9)-(10) split as follows:

$$
\begin{aligned}
\frac{1}{\delta t}\left(\hat{\mathbf{u}}^{n+1}-\mathbf{u}^{n}\right)+\hat{\mathbf{u}}^{n+1} \cdot \nabla \hat{\mathbf{u}}^{n+1}-v \Delta \hat{\mathbf{u}}^{n+1}+\gamma^{\prime} \nabla p^{n}-\mathbf{g}^{n+1} & =\mathbf{0} \\
\frac{1}{\delta t}\left(\mathbf{u}^{n+1}-\hat{\mathbf{u}}^{n+1}\right)+\nabla p^{n+1}-\gamma^{\prime} \nabla p^{n} & =\mathbf{0} \\
\nabla \cdot \mathbf{u}^{n+1} & =0
\end{aligned}
$$


where $\hat{\mathbf{u}}^{n+1}$ is an intermediate unknown, $\gamma^{\prime}$ a numerical parameter and $\mathbf{g}^{n+1}:=\mathbf{f}^{n+1}+\tau_{\mathrm{cg}} \mathbf{u}^{n} \cdot \nabla \mathbf{R}^{n}$. We will proceed here very formally, assuming that the same boundary conditions can be applied for $\hat{\mathbf{u}}^{n+1}$ and $\mathbf{u}^{n+1}$. In principle, it is well known that the continuous problem is not well posed in this case, but this is becoming a common practice in the discrete setting (see Reference [15] for further discussion).

The splitting error introduced in (13)-(15) comes from the fact that the viscous and the convective terms are computed with $\hat{\mathbf{u}}^{n+1}$ instead of $\mathbf{u}^{n+1}$. This error is of second order if $\gamma^{\prime}=1$ and of first order otherwise.

It is interesting to eliminate the end-of-step velocity $\mathbf{u}^{n}$ from (13) using (14) evaluated at time step $n$, and also $\mathbf{u}^{n+1}$ from (15) using (14) at time step $n+1$. This yields

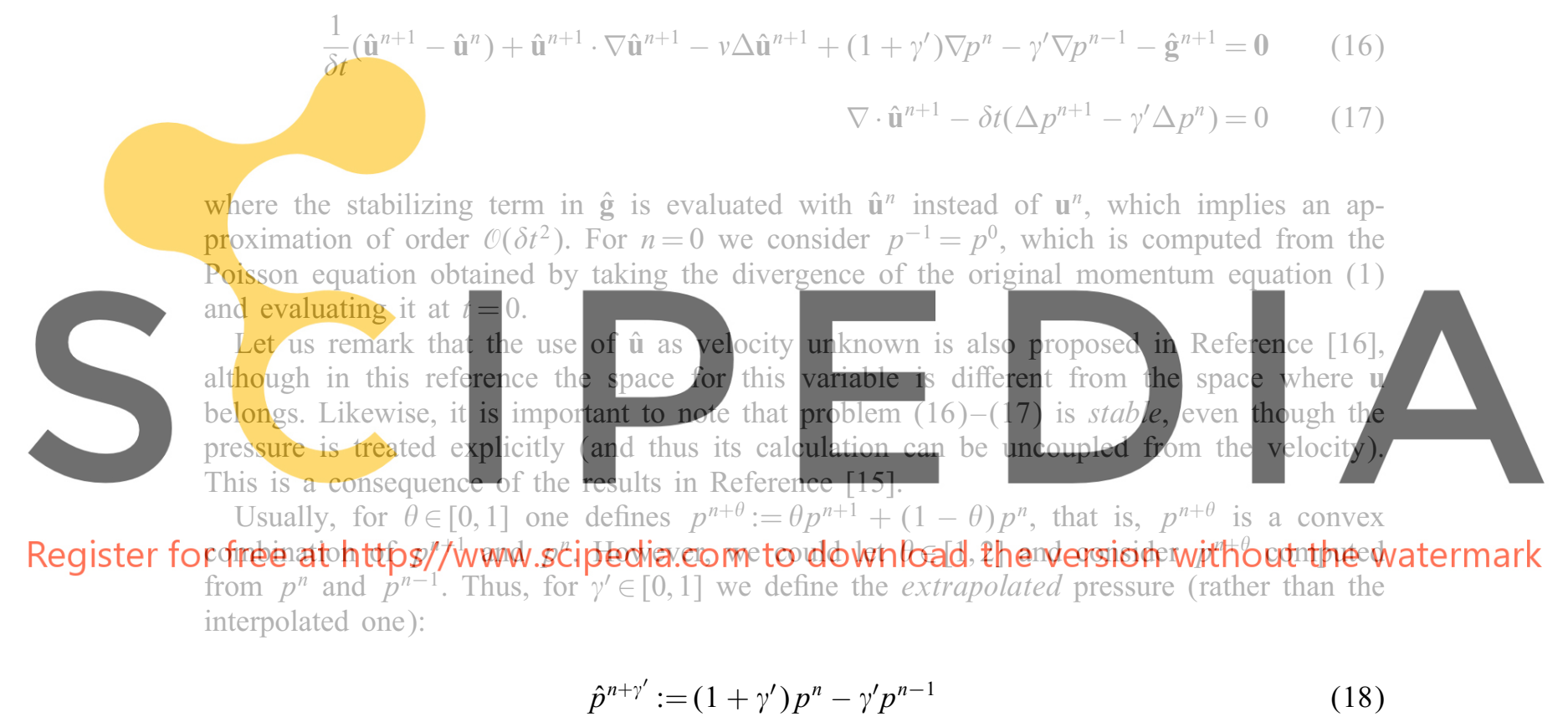

Let us define the parameter

$$
\tau_{\mathrm{s}}:=\delta t\left(1-\gamma^{\prime}\right)
$$

It allows us to replace the continuity equation by

$$
\nabla \cdot \hat{\mathbf{u}}^{n+1}-\tau_{\mathrm{s}} \Delta p^{n+1}=0
$$

which differs from (17) with a term of order $\mathcal{O}\left(\delta t^{2}\right)$.

Let $V_{h}$ be the velocity finite element space, incorporating the Dirichlet boundary conditions, and $Q_{h}$ the pressure space. As it is usual in the case of fractional step methods, the natural pressure boundary condition is zero normal derivative, which is a consequence of (14) and the fact that $\mathbf{u}$ and $\hat{\mathbf{u}}$ satisfy the same boundary conditions. 
We will also assume that $\mathbf{R}^{n}$ defined in (12) vanishes on the boundary (it is the 'residual' of the stationary equation). This, together with the fact that $\nabla \cdot \mathbf{u}^{n}=0$, allows us to integrate by parts the term coming from the discretization along the characteristics as

$$
-\int_{\Omega} \tau_{\mathrm{cg}} \mathbf{v} \cdot\left(\mathbf{u}^{n} \cdot \nabla \mathbf{R}^{n}\right) \mathrm{d} \Omega=\int_{\Omega} \tau_{\mathrm{cg}}\left(\mathbf{u}^{n} \cdot \nabla \mathbf{v}\right) \cdot \mathbf{R}^{n} \mathrm{~d} \Omega
$$

for any test function $\mathbf{v}$. Using this, definition (18), and taking the unknowns and test functions in the finite element space, the discrete weak form of problem (16)-(20) is: find $\hat{\mathbf{u}}_{h}^{n+1} \in V_{h}$ and $p_{h}^{n+1} \in Q_{h}$ such that

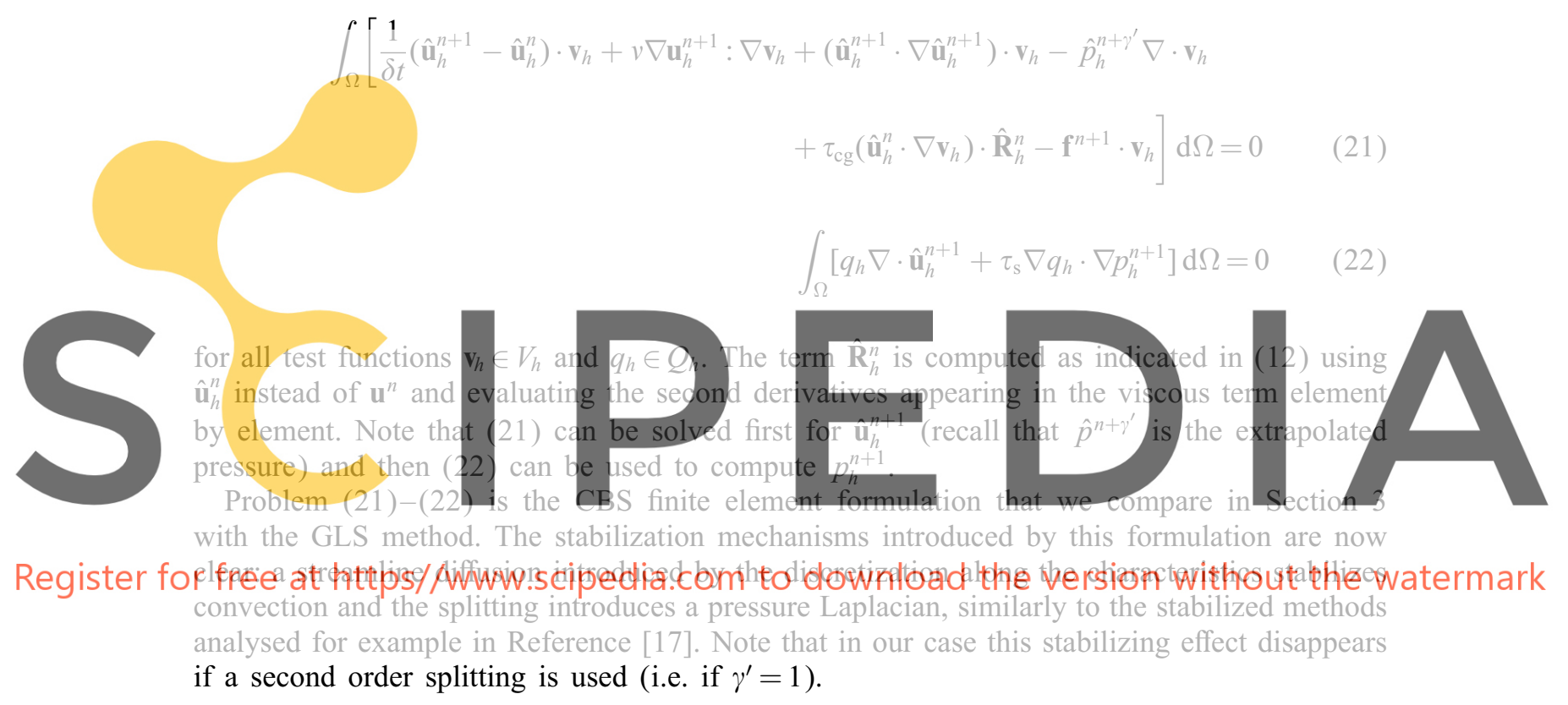

\section{THE GALERKIN/LEAST-SQUARES (GLS) METHOD}

Contrary to the CBS method, the GLS formulation deals with convection and pressure stabilization using the same approach. The original idea was to add a least-squares form of the residual within each element to the terms of the standard Galerkin formulation $[5,6]$. However, we prefer to start from a different concept, that is, the approximation of the main unknowns by the usual finite element components and an additional subgrid scale term which enhances stability of the resulting problem. This idea is proposed in Reference [18], although it is inherent in other numerical formulations. Our presentation differs from the one described in this reference, especially in the approximation of the subgrid scale (it is an extension of the method presented in Reference [19]). This will allow us to derive a formulation very similar to the original GLS (identical for linear elements) and to establish a connection with the CBS method. 
Let us start by considering the weak form of the problem written in a space-time slab $\Omega \times] t_{n}, t_{n+1}[$. It consists of finding $\mathbf{u}$ and $p$, now functions of space and time, such that

$$
\begin{aligned}
& \int_{t^{n}}^{t^{n+1}} \int_{\Omega}\left[\partial_{t} \mathbf{u} \cdot \mathbf{v}+v \nabla \mathbf{u}: \nabla \mathbf{v}+(\mathbf{u} \cdot \nabla \mathbf{u}) \cdot \mathbf{v}-p \nabla \cdot \mathbf{v}+q \nabla \cdot \mathbf{u}-\mathbf{f} \cdot \mathbf{v}\right] \mathrm{d} \Omega \mathrm{d} t \\
& \quad+\int_{\Omega}\left[\mathbf{u}\left(t_{n}^{+}\right)-\mathbf{u}\left(t_{n}^{-}\right)\right] \cdot \mathbf{v} \mathrm{d} \Omega=0
\end{aligned}
$$

for all test functions $\mathbf{v}$ and $q$, now also time dependent. We allow $\mathbf{u}$ to be discontinuous between time slabs and, in particular, the notation involved in the last term of (23) is $\mathbf{u}\left(t_{n}^{+,-}\right)=\lim _{\varepsilon \rightarrow 0^{+}-} \mathbf{u}\left(t_{n}+\varepsilon\right)$. This term imposes weakly the continuity of the velocity at $t_{n}$ (and the initial condition when $n=0$ ). Let us also remark that we have written the momentum and the continuity equation as a single variational equation.

The discrete problem is obtained by approximating $\mathbf{u}$ and $p$. If $\mathbf{u}_{h}$ and $p_{h}$ are the finite element unknowns, we approximate $\mathbf{u} \approx \mathbf{u}_{h}+\tilde{\mathbf{u}}$ and $p \approx p_{h}$, that is, the velocity is approximated by its finite element component plus an additional term that we call subgrid scale or subscale.

As in the case of the CBS method, we will consider a simple first-order scheme. In the present situation, this is obtained by taking the velocity and pressure interpolation in time as piecewise constant. We call $\boldsymbol{u}^{n+1} \approx \mathbf{u}_{*}^{n+1}:=\mathbf{u}_{h}^{n+1}+\tilde{u}^{n+1}$ and $p^{n+1} \approx p_{h}^{n+1}$ the velocity and the pressure in the time $\mathbf{u}_{h}^{n+1}$ and $p_{h}^{n+1}$ are $\mathrm{c}$ equal velocity-pressure

The important poin the interelement boundaries, $t$ to what is commonly done, we do not assume any particular behaviour of $\tilde{\mathbf{u}}^{n+1}$ within the

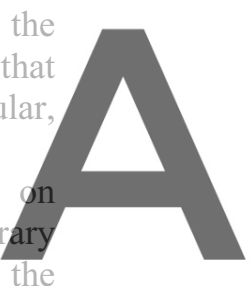
element domains. We will show later on how to approximate it.

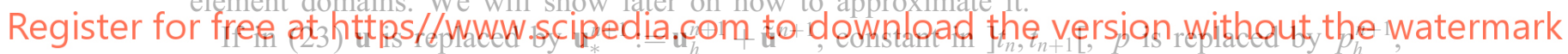
the terms involving $\tilde{\mathbf{u}}^{n+1}$ are integrated by parts, and the test functions are taken in the finite element space (also constant in the time interval considered), one gets

$$
\begin{aligned}
& \delta t \int_{\Omega}\left[v \nabla \mathbf{u}_{h}^{n+1}: \nabla \mathbf{v}_{h}+\left(\mathbf{u}_{*}^{n+1} \cdot \nabla \mathbf{u}_{h}^{n+1}\right) \cdot \mathbf{v}_{h}-p_{h}^{n+1} \nabla \cdot \mathbf{v}_{h}+q_{h} \nabla \cdot \mathbf{u}_{h}^{n+1}-\mathbf{f}^{n+1} \cdot \mathbf{v}_{h}\right] \mathrm{d} \Omega \\
& \quad+\int_{\Omega}\left[\mathbf{u}_{*}^{n+1}-\mathbf{u}_{*}^{n}\right] \cdot \mathbf{v}_{h} \mathrm{~d} \Omega-\delta t \int_{\Omega} \tilde{\mathbf{u}}^{n+1} \cdot\left(v \Delta_{h} \mathbf{v}_{h}+\mathbf{u}_{*}^{n+1} \cdot \nabla \mathbf{v}_{h}+\nabla q_{h}\right) \mathrm{d} \Omega=0
\end{aligned}
$$

where the notation $\Delta_{h}$ is used to indicate that the Laplacian needs to be evaluated element by element and $\mathbf{f}^{n+1}$ needs to be understood as the average of $\mathbf{f}$ in $] t_{n}, t_{n+1}$ [ (this option could also be used in the preceding section). Equation (24) must hold for all test functions $\mathbf{v}_{h}$ and $q_{h}$ in their corresponding finite element spaces. It is important to note that the advection velocity in (24) is $\mathbf{u}_{*}^{n+1}$ and also that the continuity between time slabs needs to be imposed in terms of this velocity.

The equation for the subscales $\tilde{\mathbf{u}}^{n+1}$ is obtained by taking the velocity test function in (23) in its space and $q=0$. The result is that, within each element [19]:

$$
\delta_{t} \mathbf{u}_{*}^{n}+\mathbf{u}_{*}^{n+1} \cdot \nabla \tilde{\mathbf{u}}^{n+1}-v \Delta \tilde{\mathbf{u}}^{n+1}=\mathbf{f}^{n+1}-\left(-v \Delta \mathbf{u}_{h}^{n+1}+\mathbf{u}_{*}^{n+1} \cdot \nabla \mathbf{u}_{h}^{n+1}+\nabla p_{h}^{n+1}\right)
$$


where $\delta_{t} \mathbf{u}_{*}^{n}:=\left(\mathbf{u}_{*}^{n+1}-\mathbf{u}_{*}^{n}\right) / \delta$. This equation states that $\mathbf{u}_{*}^{n+1}, p_{h}^{n+1}$ has to be solution of the Navier-Stokes equations within each element (and considering a piecewise constant time interpolation).

The next step would be to model (25). As it is shown in Reference [22], this is possible. However, our intention here is to recover a well-known GLS-type formulation, and for this we need to make two additional assumptions. These are to take the advection velocity $\mathbf{u}_{h}^{n+1}$ instead of $\mathbf{u}_{*}^{n+1}$ in both (24) and (25) and to neglect the first term in the latter. Even if this is done, it is obvious that (25) cannot be solved exactly. In Section 5 it will be shown in which sense we may approximate

$$
\tilde{\mathbf{u}}^{n+1}=\tau_{\mathrm{gls}}\left[\mathbf{f}^{n+1}-\left(-v \Delta \mathbf{u}_{h}^{n+1}+\mathbf{u}_{h}^{n+1} \cdot \nabla \mathbf{u}_{h}^{n+1}+\nabla p_{h}^{n+1}\right)\right]=-\tau_{\mathrm{gls}} \mathbf{R}_{h}^{n+1}
$$

where $\tau_{\mathrm{gls}}$ is a numerical parameter for which an expression is also proposed in Section 5, and $\mathbf{R}_{h}^{n+1}$ is the residual defined in (12) evaluated with $\mathbf{u}_{h}^{n+1}$ (and the second derivatives computed element by element).

With all the approximations introduced heretofore, the final discrete problem to be solved for $\mathbf{u}_{h}^{n+1}$ and $p_{h}^{n+1}$ is
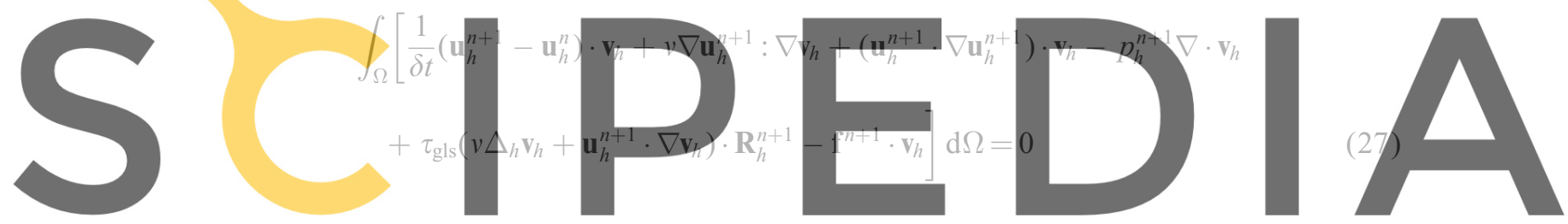

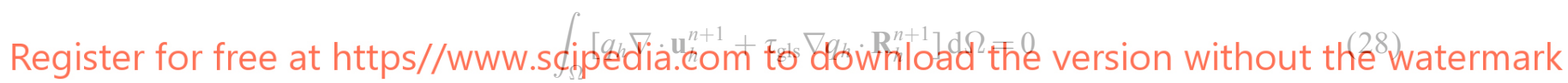

which is obtained from (24) by dividing this equation by $\delta t$, taking $\mathbf{u}_{h}^{n+1}$ as advection velocity, using (26) as approximation to $\tilde{\mathbf{u}}^{n+1}$ and splitting the momentum and continuity equations (taking in (24) $q_{h}=0$ and $\mathbf{v}_{h}=\mathbf{0}$, respectively). Except for the sign of $v \Delta_{h} \mathbf{v}_{h}$, with all the assumptions and approximations introduced we have arrived to the most familiar form of the GLS formulation (for a first-order time approximation).

\section{COMPARISON OF CBS AND GLS}

We are now in a position to compare the CBS and GLS formulations, given respectively by Equations (21)-(22) and (27)-(28). The differences between both methods are the following:

- The pressure gradient in the CBS method is extrapolated from values of the pressure in previous time steps. This does not affect the stabilization mechanism of the method, but only its implementation: it is possible to solve for the velocity first and to compute the pressure afterwards. 
- The stabilization of the convective term is achieved in both cases through the introduction of streamline diffusion. The stabilizing terms are

$$
\begin{array}{ll}
\text { CBS: } & \tau_{\mathrm{cg}}\left(\hat{\mathbf{u}}_{h}^{n} \cdot \nabla \mathbf{v}_{h}\right) \cdot \hat{\mathbf{R}}_{h}^{n} \\
\mathrm{GLS}: & \tau_{\mathrm{gls}}\left(v \Delta_{h} \mathbf{v}_{h}+\mathbf{u}_{h}^{n+1} \cdot \nabla \mathbf{v}_{h}\right) \cdot \mathbf{R}_{h}^{n+1}
\end{array}
$$

Except for the time level where the residual is evaluated (which is irrelevant for our discussion) and the viscous operator applied to the test function for the GLS method (which is zero for linear elements) we see that both methods introduce the same amount of streamline diffusion if we identify $\tau_{\mathrm{gls}}=\tau_{\mathrm{cg}}:=(2 \gamma-1) \delta t / 2$. Remember that $\gamma$ defines the position along the characteristics at which the equations are discretized in time (see Figure 1).

- Pressure is stabilized in both cases through the introduction of a pressure-Laplacian. For the CBS method, this is what is directly introduced, whereas for the GLS formulation this term is part of the weighting of the element residual:

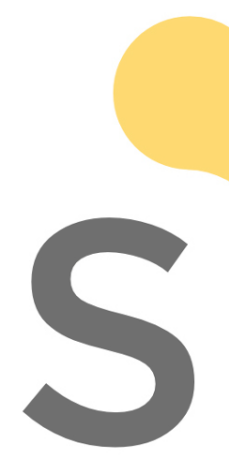

CBS: $\tau_{\mathrm{s}} \nabla q_{h} \cdot \nabla p_{h}^{n+1}$

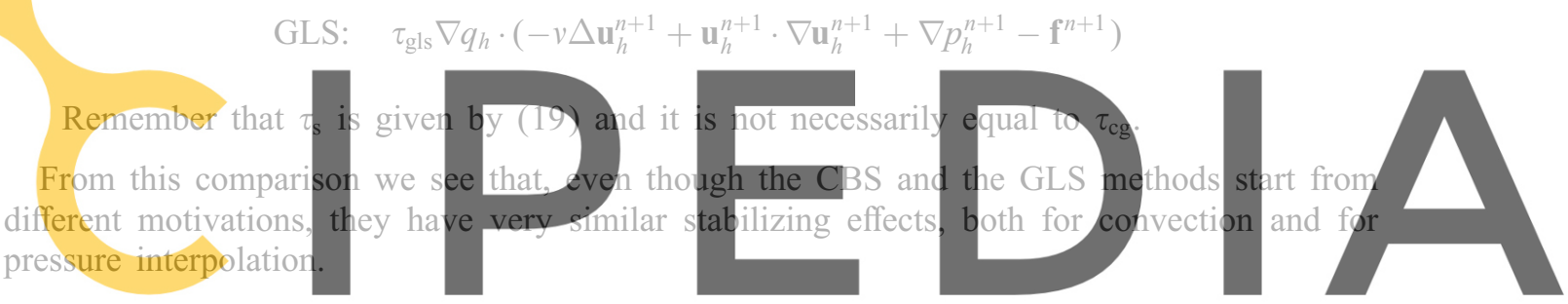

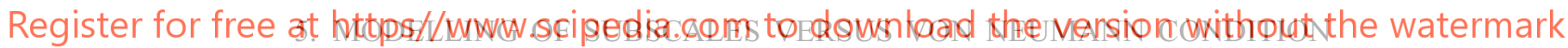

From the comparison of the stabilizing terms of the CBS and the GLS formulations it is clear that the parameters $\tau_{\mathrm{cg}}, \tau_{\mathrm{s}}$ and $\tau_{\mathrm{gls}}$ need to be related, in some sense, to the time step size $\delta t$. Moreover, experience dictates that their optimal values are close to what would be the critical time step of the explicit Euler scheme for a convection-diffusion equation (taking $v$ as diffusion coefficient) using one of the most common stabilized formulations, such as SUPG [23] or Taylor-Galerkin [24]. In this section we use a heuristic argument to show that this fact can be formally justified.

Our starting point will be to give a closed-form expression for the subgrid scales solution of problem (25) assuming, as it has been said before, that $\delta_{t} \mathbf{u}_{*}^{n}$ can be neglected. We do not want to solve (25) exactly, but rather to model it, that is to say, to propose an expression for it whose validity has to be confirmed by numerical experiments and/or analysis.

Let us assume that $\mathbf{u}_{*}^{n+1}$ in (25) is given and let us call it $\mathbf{a}$. Dropping the superscript $n+1$ for notational convenience, $\tilde{\mathbf{u}}$ is a solution of

$$
-v \Delta \tilde{\mathbf{u}}+\mathbf{a} \cdot \nabla \tilde{\mathbf{u}}=-\mathbf{R}_{h}
$$

within each element $\Omega^{e}$. We want to understand in which sense $\tilde{\mathbf{u}}(\mathbf{x}) \approx-\tau_{\mathrm{gls}} \mathbf{R}_{h}(\mathbf{x})$ and to give an expression for $\tau_{\mathrm{gls}}$. 
Let us consider the following Fourier transform defined on each element domain $\Omega^{e}$ :

$$
\hat{g}(\mathbf{k}):=\int_{\Omega^{e}} \mathrm{e}^{-\mathrm{i}(\mathbf{k} \cdot \mathbf{x} / h)} g(\mathbf{x}) \mathrm{d} \Omega_{x}
$$

where $\mathbf{k}=\left(k_{1}, k_{2}, k_{3}\right)$ is the dimensionless wave number (in 3D) and $h$ is the diameter of element $\Omega^{e}$. If $n_{j}$ is the $j$ th component of the normal exterior to $\Omega^{e}$, it is easily checked that

$$
\frac{\widehat{\partial g}}{\partial x_{j}}(\mathbf{k})=\int_{\partial \Omega^{e}} n_{j} \mathrm{e}^{-\mathrm{i}(\mathbf{k} \cdot \mathbf{x} / h)} g(\mathbf{x}) \mathrm{d} \Gamma_{x}+\mathrm{i} \frac{k_{j}}{h} \hat{g}(\mathbf{k})
$$

We assume that the subscales only contain high wave numbers, and thus

$$
\frac{\widehat{\partial \tilde{\mathbf{u}}}}{\partial x_{j}}(\mathbf{k}) \approx \mathrm{i} \frac{k_{j}}{h} \hat{\tilde{\mathbf{u}}}(\mathbf{k}), \quad \frac{\widehat{\partial^{2} \tilde{\mathbf{u}}}}{\partial x_{i} \partial x_{j}}(\mathbf{k}) \approx-\frac{k_{i} k_{j}}{h^{2}} \hat{\tilde{\mathbf{u}}}(\mathbf{k})
$$

Note that all the results valid for Fourier transforms of functions of rapid decay in $\mathbb{R}^{d}$ will apply to $\hat{\tilde{\mathbf{u}}}$.

Equation (30) allows us to obtain an expression for $\tau_{\mathrm{gls}}$. Indeed, if we take the Fourier transform of (29) we have

$$
\hat{\tilde{\mathbf{u}}}(\mathbf{k}) \approx \mathscr{T}(\mathbf{k}) \hat{r}(\mathbf{k}), \quad \mathscr{T}(\mathbf{k}):=\left(v \frac{|\mathbf{k}|^{2}}{h^{2}}+\mathrm{i} \frac{\mathbf{a} \cdot \mathbf{k}}{h}\right)^{-1}
$$

Plancherel's formula and the mean value theorem imply

$$
\begin{aligned}
\|\tilde{\mathbf{u}}\|_{L^{2}\left(\Omega^{e}\right)}^{2} & \approx \frac{1}{(2 \pi)^{d}}\|\hat{\tilde{\mathbf{u}}}\|_{L^{2}\left(\mathbb{R}^{d}\right)}^{2} \\
& \approx \frac{1}{(2 \pi)^{d}} \int_{\mathbb{R}^{d}}|\mathscr{T}(\mathbf{k})|^{2}\left|\hat{\mathbf{R}}_{h}(\mathbf{k})\right|^{2} \mathrm{~d} k \\
& =\frac{1}{(2 \pi)^{d}}\left|\mathscr{T}\left(\mathbf{k}_{0}\right)\right|^{2} \int_{\mathbb{R}^{d}}\left|\hat{\mathbf{R}}_{h}(\mathbf{k})\right|^{2} \mathrm{~d} k \\
& =\left|\mathscr{T}\left(\mathbf{k}_{0}\right)\right|^{2}\left\|\mathbf{R}_{h}\right\|_{L^{2}\left(\Omega^{e}\right)}^{2}
\end{aligned}
$$

for a certain $\mathbf{k}_{0}$. If we identify $\tau_{\mathrm{gls}}$ with $\left|\mathscr{T}\left(\mathbf{k}_{0}\right)\right|$, it allows us to conclude that if we take

$$
\tau_{\mathrm{gls}}=\left[\left(c_{1} \frac{v}{h^{2}}\right)^{2}+\left(c_{2} \frac{|\mathbf{a}|}{h}\right)^{2}\right]^{-1 / 2}
$$

there exist values of $c_{1}$ and $c_{2}$ independent of $h, v$ and $|\mathbf{a}|$ for which both $\tilde{\mathbf{u}}$ and $-\tau_{\mathrm{gls}} \mathbf{R}_{h}$ have (approximately) the same $L^{2}$-norm on element $\Omega^{e}$.

Expression (31) behaves asymptotically as the critical time step of the explicit Euler scheme for the convection-diffusion equation using a stabilized method [1]. For simplicity, let us consider the scalar case and the stabilization through the introduction of artificial numerical 
diffusion, so that the equation that needs to be discretized in space using the standard Galerkin method is

$$
\frac{1}{\delta t}\left(u^{n+1}-u^{n}\right)-v_{\text {num }} \Delta u^{n}+\mathbf{a} \cdot \nabla u^{n}=f
$$

with

$$
v_{\text {num }}:=v+c|\mathbf{a}| h
$$

where $h$ is an element diameter defined a priori and $c$ is a constant that determines the amount of artificial diffusion.

Let us show that (a particular version of) the von Neumann stability analysis leads to a critical time step which has the same asymptotic behaviour as (31). To compute this time step, one has to build up a perturbation function and see that it is necessarily damped out by the numerical scheme. This perturbation function must be a solution of the homogeneous problem. We construct it as solution of

$$
\frac{1}{\delta t}\left(e^{n+1}-e^{n}\right)-v_{\text {num }} \Delta e^{n}+\mathbf{a} \cdot \nabla e^{n}=0
$$

within each element $\Omega^{e}$, with $e^{n+1}=0$ on $\partial \Omega^{e}$. This particular perturbation propagates in time according to the operational relationship

$$
e^{n+1}=\left(1+\delta t v_{\text {num }} \Delta-\delta t \mathbf{a} \cdot \nabla\right) e^{n}
$$

Taking the Fourier transform defined above and assuming again that $e^{n+1}$ contains only high wave numbers, we obtain

$$
\left\|e^{n+1}\right\|^{2} \approx\left|1-\delta t v_{\text {num }} \frac{\left|\mathbf{k}_{0}\right|^{2}}{h^{2}}-\delta t \mathbf{i} \frac{\mathbf{a} \cdot \mathbf{k}_{0}}{h}\right|^{2}\left\|e^{n}\right\|^{2}
$$

where the notation involved is the same as before. A simple calculation shows that a sufficient condition for $\left\|e^{n+1}\right\| \leqslant\left\|e^{n}\right\|$ to hold is that

$$
\delta t \leqslant \delta t_{\text {crit }}:=\left(c_{1} \frac{v}{h^{2}}+c_{2} \frac{|\mathbf{a}|}{h}\right)^{-1}
$$

where now

$$
c_{1}=\frac{\left|\mathbf{k}_{0}\right|^{2}}{2}\left(1+\frac{1}{c^{2}\left|\mathbf{k}_{0}\right|^{2}}\right), \quad c_{2}=c c_{1}
$$

and $c$ is the constant in (32). Clearly, $\delta t_{\text {crit }}$ given by (33) behaves asymptotically like $\tau_{\text {gls }}$ given by (31) in terms of $h, v$ and $|\mathbf{a}|$.

This development has shown that the ideas used to model the subscales of the GLS formulation (vanishing on the interelement boundaries, dominance of the high wave number components, equation for the norms of the Fourier transform of (29), identification of the algorithmic constants with wave numbers) can be applied to the classical von Neumann analysis as well. 


\section{CONCLUSIONS}

In this paper, we have compared the CBS and the GLS formulations to solve the incompressible Navier-Stokes equations. The presentation has highlighted the stabilization mechanisms of both, showing that they are in fact very similar, both for the stabilization of the convective term and the pressure interpolation.

It has also been shown that the stabilizing parameters of the GLS method are related to the critical time step size of stabilized formulations for convection-diffusion problems. For the CBS method the stabilizing terms are directly proportional to the time step size.

As a by-product of our discussion, it has been justified why in the CBS formulation the factors that appear in the stabilizing terms are not necessarily $\delta t$. For the streamline diffusion term, this factor depends on the position along the characteristics at which the equations are discretized, whereas for the pressure stabilizing term it depends on the fraction of pressure gradient kept in the prediction step (13).

\section{REFERENCES}

1. Zienkiewicz OC, Taylor RL. The Finite Element Method, Fluid Mechanics, vol. 3 (5th edn). ButterworthHeinemann: Stoneham, MA, 2000.

2. Codina R, Vázquez M, Zienkiewicz OC. A general algorithm for compressible and incompressible flow-Part III. The semi-implicit form. International Journal for Numerical Methods in Fluids 1998; 27:13-32.

3. Zienkiewicz OC, Codina R. A general algorithm for compressible and incompressible flow-Part I. The split, characteristic-based scheme. International Journal for Numerical Methods in Fluids 1995; 20:869-885.

4. Zienkiewicz OC, Nithiarasu P, Codina R, Vázquez M, Ortiz P. The characteristic based split procedure: an efficient and accurate algorithm for fluid problems. International Journal for Numerical Methods in Fluids 1999; 31:359-392.

5. Hughes TJR, Franca LP, Hulbert GM. A new finite element formulation for computational fluid dynamics: VIII. The Galerkin/least-squares method for advective-diffusive equations. Computer Methods in Applied Mechanics and Engineering 1989; 73:173-189.

6. Franca LP, Hughes TJR. Convergence analyses of Galerkin least-squares methods for advective-diffusive forms of the Stokes and incompressible Navier-Stokes equations. Computer Methods in Applied Mechanics and Engineering 1993; 105:285-298.

7. Shakib F, Hughes TJR. A new finite element formulation for computational fluid dynamics: IX. Fourier analysis of space-time Galerkin/least-squares algorithms. Computer Methods in Applied Mechanics and Engineering 1991; 87:35-58.

8. Codina R. Numerical solution of the incompressible Navier-Stokes equations with Coriolis forces based on the discretization of the total time derivative. Journal of Computational Physics 1999; 148:467-496.

9. Douglas J, Russel T. Numerical methods for convection dominated problems based on combining the method of characteristics with finite elements or finite difference procedures. SIAM Journal on Numerical Analysis 1982; 19:871-885.

10. Pironneau O. On the transport-diffusion algorithm and its application to the Navier-Stokes equations. Numerische Mathematik 1982; 38:309-332.

11. Löhner R, Morgan K, Zienkiewicz OC. The solution of non-linear hyperbolic equations system by the finite element method. International Journal for Numerical Methods in Fluids 1984; 4:1043-1063.

12. Chorin AJ. Numerical solution of the Navier-Stokes equations. Mathematics of Computation 1968; 22: 745-762.

13. Temam R. Sur l'approximation de la solution des équations de Navier-Stokes par la méthode des pas fractionaires (I). Archives for Rational Mechanics and Analysis 1969; 32:135-153.

14. Rannacher R. On Chorin's Projection Method for Incompressible Navier-Stokes Equations, Lecture Notes in Mathematics, Vol. 1530. Springer: Berlin, 1992; 167-183.

15. Codina R. Pressure stability in fractional step finite element methods for incompressible flows. Journal of Computational Physics 2001; 170:112-140.

16. Guermond JL, Quartapelle L. Calculation of incompressible flows by an unconditionally stable FEM algorithm. Journal of Computational Physics 1997; 132:12-33.

17. Brezzi F, Douglas J. Stabilized mixed methods for the Stokes problem. Numerische Mathematik 1988; 53: $225-235$. 
18. Hughes TJR, Feijóo GR, Mazzei L, Quincy JB. The variational multiscale method—a paradigm for computational mechanics. Computer Methods in Applied Mechanics and Engineering 1998; 166:3-24.

19. Codina R. A stabilized finite element method for generalized stationary incompressible flows. Computer Methods in Applied Mechanics and Engineering 2001; 190:2681-2706.

20. Baiocchi C, Brezzi F, Franca LP. Virtual bubbles and Galerkin/least-squares type methods (Ga.L.S). Computer Methods in Applied Mechanics and Engineering 1993; 105:125-141.

21. Brezzi F, Franca LP, Hughes TJR, Russo A. $b=\int g$. Computer Methods in Applied Mechanics and Engineering 1997; 145:329-339.

22. Codina R. Stabilized finite element approximation of transient incompressible flows using orthogonal subscales. Computer Methods in Applied Mechanics and Engineering, submitted.

23. Brooks AN, Hughes TJR. Streamline upwind/Petrov-Galerkin formulations for convection dominated flows with particular emphasis on the incompressible Navier-Stokes equation. Computer Methods in Applied Mechanics and Engineering 1982; 32:199-259.

24. Donea J. A Taylor-Galerkin method for convection transport problems. International Journal for Numerical Methods in Engineering 1984; 20:101-119. 